\title{
ON THE SYMMETRY AND BOUNDED CLOSURE OF LOCALLY CONVEX SPACES
}

\author{
BY \\ WILLIAM F. DONOGHUE, JR. AND KENNAN T. SMITH
}

Preface. For some time it has been recognized that the properties of symmetry and bounded closure are of cardinal importance in the theory of locally convex spaces. The purpose of this paper is to discuss symmetry and bounded closure with the aid of a new topology, and to discuss the new topology itself.

The introduction includes the preliminaries to the rest of the paper. The account of the basic theory of locally convex spaces is fairly complete, but brief. We have given proofs only when they represent some contribution. As the references indicate, most of the theorems of the introduction can be found in the literature.

In $\$ 1$ we introduce the new topology, called the $G F$ topology, and develop the theory of the $G F$ space. A space is a $G F$ space if and only if it is symmetric and boundedly closed. Study of the $G F$ topology on duals leads to information about interior maps and quotient spaces, information which is not obvious from direct consideration of symmetry and bounded closure. We conclude the section with several examples.

In view of the general results of the first section it is important to know what spaces are $G F$ spaces. In $\$ 2$ we take up this question for direct products and their duals, and also the questions of symmetry and bounded closure. $\$ 3$ serves the same purpose for continuous function spaces. Finally in $\$ 4$ we discuss metric and $L F$ spaces and their duals.

The influence of the works of G. W. Mackey, J. Dieudonné, and L. Schwartz is apparent throughout the paper, and it is to these authors that we owe our interest in locally convex spaces. The terminology used in the paper is almost exclusively that employed in the works of Dieudonné, Schwartz, and Bourbaki. For the convenience of the reader we list the exceptions. We distinguish between weak and weak-star topologies; our total bounded is the French precompact and corresponds to the definition given in [14]; we owe the term relatively strong to Mackey [13]; where the French authors use filters we make use of nets, as in Kelley [10]; and for a uniform structure topology the natural definition of a Cauchy net leads to a concept of completeness which is equivalent to the French term and stronger than that given in [14]. There are no French equivalents for symmetry and bounded closure.

Received by the editors December 9, 1951. 
Most of the work on the paper was done at the University of Wisconsin with the encouragement and valuable counsel of Professors Laurence C. Young and William F. Eberlein. The authors are deeply grateful to Professors Young and Eberlein.

Introduction. Closely associated with any linear space $E$ over the complex numbers are the linear forms on $E$, the functions $f(x)$ from $E$ to the complex numbers satisfying, for $x$ and $y$ in $E$ and $\lambda$ a complex number, $f(x+y)$ $=f(x)+f(y)$ and $f(\lambda x)=\lambda f(x)$. When addition and scalar multiplication are defined in the obvious fashion, the class of linear forms becomes a linear space, which we call the algebraic dual of $E$ and denote by $\bar{E}$. If $E$ is a topological space as well as a linear space, those linear forms which are contiruous are of particular importance. The set of continuous linear forms is a subspace of $\bar{E}$, which we call the dual of $E$ and denote by $E^{\prime}$.

Let $E$ be a linear space and let $F$ be a subspace of its algebraic dual which contains for each $x \neq 0$ in $E$ an $f$ such that $f(x) \neq 0$. Then $E$ is a subspace of the algebraic dual of $F$ with the same property. To exhibit the duality between $E$ and $F$ we write $(f, x)$ rather than $f(x)$. The polar in $F$ of a set $A$ in $E$, written $A^{\circ}$, is the set of all $f$ in $F$ such that if $x$ is in $A$, then $|(f, x)| \leqq 1$. The polar in $E$ of a set $A$ in $F$ is defined similarly. $F$ defines on $E$ a natural topology called the weak topology and represented by the symbol $W(E, F)$. Its neighborhoods of the point 0 are the polars in $E$ of finite sets in $F$. Its neighborhoods of the point $x$ are the sets $x+V$, where $V$ is a neighborhood of 0 . In the same way $E$ defines on $F$ a natural topology called the weak-star topology and represented by the symbol $W(F, E)$.

The weak topology on $E$ is defined by neighborhoods of the origin; each neighborhood of the origin is convex $(V+V \subseteq 2 V)$ and circled $\left(e^{i \lambda} V \subseteq V\right)$, and the operations of addition and scalar multiplication are jointly continuous. Any topology on $E$ having these properties is called a locally convex topology. A linear space with a locally convex topology is a locally convex space.

Let $E$ with topology $T$ be a locally convex space. We indicate that $V$ is a neighborhood of the origin in the topology $T$ by saying that $V$ is in $T$ or that $V$ is a $T$ neighborhood or just that $V$ is a neighborhood. The semi-norm associated with the neighborhood $V$ is the function $v(x)=\inf \{|\lambda|:(1 / \lambda) x$ in $V\}$. The closure of $V$ is the set where $v(x) \leqq 1$, so that $v$ is continuous, and the topology $T$ can be described by specifying its semi-norms as well as by specifying its neighborhoods. Every semi-norm has the properties $v(x+y) \leqq v(x)$ $+v(y)$ and $v(\lambda x)=|\lambda| v(x)$. A linear form $f$ on $E$ is continuous if and only if it is bounded on a neighborhood, and hence if and only if it satisfies $|(f, x)|$ $\leqq v(x)$ for some semi-norm $v$. With the notion of semi-norm it is easy to prove the following theorem.

Theorem 1. If $A$ is a closed convex circled subset of $E$ and $y$ is in $E-A$, then there is an $f$ in $E^{\prime}$ such that $(f, y)>1$ and $|(f, x)| \leqq 1$ for every $x$ in $A[7]$. 
Any theorem of this type which describes the separation of a point and a convex set by a hyperplane is called a Hahn-Banach theorem. The HahnBanach theorem implies, in particular, that $E^{\prime}$ has the property previously required of $F$ : for each $x \neq 0$ in $E$ it contains an $f$ such that $(f, x) \neq 0$. A HahnBanach theorem is also valid for $E^{\prime}$ in the weak-star topology.

Theorem 2. If $A$ is a weak-star closed convex circled subset of $E^{\prime}$ and $g$ is in $E^{\prime}-A$, then there is an $x$ in $E$ such that $(g, x)>1$ and $|(f, x)| \leqq 1$ for every $f$ in $A[5 ; 7]$.

A set $A$ in $E$ is $T$ bounded, or simply bounded, if to each $T$ neighborhood $V$ corresponds a number $\lambda$ such that $\lambda A \subseteq V$. A linear form on $E$ is bounded if it is bounded on each bounded set. The bounded sets in $E$ are used to define another topology on $E^{\prime}$. It is called the ordinary topology, is labeled $T^{\prime}$, and its neighborhoods are the polars of the $T$ bounded sets. Unqualified topological terms such as bounded, closed, continuous refer either to the given topology $T$ of $E$ or to the ordinary topology $T^{\prime}$ of $E^{\prime}$. Since $W\left(E, E^{\prime}\right)$, $W\left(E^{\prime}, E\right), T$, and $T^{\prime}$ are all locally convex topologies, it is proper to speak of boundedness in any of them.

THEOREM 3. Weak bounded and $T$ bounded are the same. If $E$ is sequentially complete, weak-star bounded and $T^{\prime}$ bounded are the same $[12 ; 13]$.

Every locally convex topology is a uniform structure and the usual definition of total bounded is employed.

THEOREM 4. In weak or weak-star topologies bounded and total bounded are the same [7].

Using Theorem 4 it is easy to prove the following theorem.

THEOREM 5. If $V$ is a neighborhood in $E$, then $V^{\circ}$ is weak-star compact $[1 ; 5]$.

It is a consequence of the Hahn-Banach theorem that, for any set $A$ in $E\left(E^{\prime}\right), A^{\circ \circ}$ is the smallest closed (weak-star closed) convex circled set containing $A[7]$. It follows that the topology $T$, as well as all of the others which have been mentioned, can be defined by taking as neighborhoods the polars of certain convex circled and weak or weak-star compact sets. The polars of all convex circled weak-star compact sets form the neighborhoods for a locally convex topology on $E$ which we call the relatively strong topology and write $T\left(E, E^{\prime}\right) . T\left(E, E^{\prime}\right)$ is clearly stronger than $T$, and its precise relation to the several topologies on $E$ is given by the fundamental Mackey-Arens theorem $[2 ; 12 ; 13]$.

THEOREм 6. $T\left(E, E^{\prime}\right)$ is the strongest locally convex topology on $E$ such that $E^{\prime}$ is the dual of $E$. 
As the known proofs of the Mackey-Arens theorem are complicated, we offer a simple proof of our own. In view of the preceding remarks, if $T\left(E, E^{\prime}\right)$ does yield $E^{\prime}$ as the dual of $E$, it is the strongest topology doing so.

A convex circled weak-star compact subset of $E^{\prime}$ is a convex circled weakstar closed subset of $\bar{E}$. Thus if $C$ is such a set, $C$ is the polar in $\bar{E}$ of $C^{\circ}$, and any linear form which is bounded on $C^{\circ}$ is a scalar multiple of an element in $C$, and hence lies in $E^{\prime}$.

It is a simple matter to deduce from the Mackey-Arens theorem the following theorem.

THEOREM 7. Every convex circled weak-star compact subset of $E^{\prime}$ is bounded.

The foregoing discussion reveals that any linear space $E$ can be converted into a locally convex space having as dual a prescribed weak-star dense subspace $F$ of $\bar{E}$. Among the topologies which serve the weakest is $W(E, F)$ and the strongest is $T(E, F)$. In comparing the different topologies it is convenient to use the words "stronger than", to mean "at least as strong" and to give this meaning to the symbol $\geqq$.

Much of our discussion concerns the continuity of linear forms. We have observed that for a linear form $f$ the assertions that $f$ is bounded on a neighborhood and that $f$ is continuous are equivalent. A third equivalent assertion is that the null space of $f$ is closed.

For a linear transformation from one locally convex space to another the three assertions are not equivalent in general, but the first implies the second, which in turn implies the third. If $T$ is a linear transformation from the locally convex space $E$ into the locally convex space $F$, its adjoint $T^{*}$ is the linear transformation from $F^{\prime}$ into $\bar{E}$ defined by the equation $\left(T^{*} f, x\right)$ $=(f, T x) . T^{*}$ is a linear transformation from $F^{\prime}$ into $E^{\prime}$ if and only if $T$ is continuous when $E$ and $F$ have the weak topologies: i.e., $T$ is weak continuous. If $T$ is weak continuous, then $T^{*}$ is both continuous and weak-star continuous.

The dual of the locally convex space $E^{\prime}$ with topology $T^{\prime}$ is called the second dual of $E$ and is written $E^{\prime \prime}$. As a dual $E^{\prime \prime}$ has an ordinary topology $T^{\prime \prime}$. According to the previous definition, the polar in $E^{\prime \prime}$ of an arbitrary bounded set in $E^{\prime}$ is a neighborhood in $T^{\prime \prime}$. If the polar in $E$ of an arbitrary bounded set in $E^{\prime}$ is a neighborhood in $T$, then the space $E$ and the topology $T$ are called symmetric.

We have noted that $E$ is a subspace of $\bar{E}^{\prime}$; as the topology $T^{\prime}$ is clearly stronger than the topology $W\left(E^{\prime}, E\right), E$ is a subspace of $E^{\prime \prime}$. The identity mapping from $E$ as a locally convex space to $E$ as a subspace of $E^{\prime \prime}$ is termed the canonical mapping. As the neighborhoods in $T$ are the polars of certain convex circled weak-star compact sets in $E^{\prime}$, and as each of these is bounded, $T$ is weaker than $T^{\prime \prime}$. Clearly the two are identical if and only if $E$ is symmetric. The considerations which apply to $T$ also apply to $T\left(E, E^{\prime}\right)$, and 
$T\left(E, E^{\prime}\right)$ is weaker than $T^{\prime \prime}$. Thus if $E$ is symmetric, it has the relatively strong topology, and every bounded set in $E^{\prime}$ is contained in a convex circled weak-star compact set. Evidently the canonical mapping is continuous if and only if $E$ is symmetric.

If the canonical mapping of $E$ into $E^{\prime \prime}$ is onto, i.e., if the elements of $E$ are the only continuous linear forms on $E^{\prime}$, then clearly $T^{\prime}=T\left(E^{\prime}, E\right)$. When this is the case we say that $E$ is semi-reflexive, and it is clear that $E$ is semireflexive if and only if any bounded set in $E$ is contained in a convex circled weak compact set. The space $E$ is called reflexive when $E$ and $E^{\prime \prime}$ coincide both algebraically and topologically, that is, when $E$ is both symmetric and semi-reflexive. If $E$ is semi-reflexive, then $E^{\prime}$ is symmetric, for a bounded set in $E^{\prime \prime}$ is also a bounded set in $E$, hence its polar in $E^{\prime}$ contains a neighborhood.

A locally convex space is called a metric space if there exists a fundamental set of neighborhoods which is countable. The topology of such a space is indeed a metric topology. The metric spaces are the only locally convex spaces which have a fundamental set of neighborhoods linearly ordered under set inclusion. A metric space is called an $F$ space if it is complete.

The locally convex space $E$ is said to be boundedly closed if every bounded linear form on $E$ is continuous. We use the term bounded closure to describe the property of being boundedly closed, and not in the way it was used originally by Mackey [13].

\section{THEOREM 8. Every metric space is symmetric and boundedly closed.}

The bounded closure is established in [12]. A trivial modification of the argument establishes the symmetry. Since every $L F$ space is also symmetric [7], we have the following corollary.

CoRollary. Every semi-reflexive metric or LF space is reflexive.

We have no example of a symmetric space which is not boundedly closed. Boundedly closed spaces which are not symmetric are common, but we have:

THEOREM 9. Every boundedly closed space is symmetric in the relatively strong topology.

Proof. If $B$ is bounded in $E^{\prime}$, then $C$, the smallest weak-star closed convex circled set containing $B$, is also bounded, and hence weak-star total bounded. We show that $C$ is a weak-star complete, therefore weak-star compact, and this fact implies that $B^{\circ}=C^{\circ}$ is a relatively strong neighborhood. If $f_{\alpha}$ is a Cauchy net in $C$, the linear form $(f, x)=\lim _{\alpha}\left(f_{\alpha}, x\right)$ is bounded on bounded sets, hence is continuous.

THEOREM 10. There is at most one symmetric topology on $E$ yielding a prescribed dual. 
Proof. We have already observed that if $E$ admits a symmetric topology with dual $E^{\prime}$, then $T=T^{\prime \prime}=T\left(E, E^{\prime}\right)$.

COROLLARY. There is at most one metric topology on E yielding a prescribed dual.

We conclude this introduction with some representation theorems which are responsible for our interest in direct products and continuous function spaces.

THEOREM 11. Every locally convex space is isomorphic to a subspace of a direct product of Banach spaces [8].

Proof. If $v(x)$ is a semi-norm on $E$ and $H_{v}$ is its null space, then $E_{v}$, the completion of $E / H_{v}$ with norm $v$, is a Banach space. The transformation which maps $x$ in $E$ into the function $x(v)$, where $x(v)$ is the canonical image of $x$ in $E_{v}$, is an algebraic isomorphism between $E$ and a subspace of the direct product of the $E_{v}$. Convergence, both in the direct product and in $E$, is convergence with respect to each $v$, so that the algebraic isomorphism is also a topological isomorphism.

THEOREM 12. Every locally convex space is isomorphic to a subspace of a $C(S)$ : the continuous functions on a locally compact Hausdorff space, $S$, in the compact-open topology.

Proof. Because of the previous theorem, it is enough to show this result for a direct product of Banach spaces. By a result of Alaoglu [1] every Banach space $E_{v}$ is a closed subspace of the space of continuous functions on some compact Hausdorff space $S_{v}$. Let $S$ be the set union of the $S_{v}$, and topologize $S$ by assigning to each point the neighborhood system which it has in the $S_{v}$ to which it belongs. It follows that $S$ is a locally compact Hausdorff space, and that the direct product of the $E_{v}$ is a subspace of $C(S)$. Convergence in $C(S)$ implies convergence in each $C\left(S_{v}\right)$, hence convergence in the product. On the other hand, every compact set in $S$ is contained in the union of finitely many $S_{v}$, whence convergence in the product implies convergence in $C(S)$.

COROLlary. Every separable metric space is isomorphic to a subspace of $C(R)$, where $R$ is the real axis in the usual topology.

Proof. We argue as before, using the theorem that a separable Banach space is isomorphic to a subspace of $C([0,1])[3]$. The remainder of the argument is clear.

1. GF spaces. In 1949 in a paper entitled La dualité dans les espaces $F$ et $L F$ [7], Dieudonné and Schwartz introduced a new type of locally convex space called the $L F$ space. In this section we generalize the concept of $L F$ space. We develop a theory for the new spaces and examine the concepts of symmetry and bounded closure. We show how the new $G F$ spaces arise nat- 
urally in the study of locally convex spaces and, indeed, that many of the common spaces are $G F$ spaces.

A Dieudonné-Schwartz $L F$ space is a locally convex space $E$ whose topology is defined by an ascending sequence of $F$ spaces $E_{n}$. It is supposed that the $F$ spaces have compatible topologies and that their set union is $E$. A neighborhood of the origin in $E$ is then any convex circled set whose intersection with each $E_{n}$ is a neighborhood of the origin in $E_{n}$.

To generalize the $L F$ space we proceed as follows. Let $E$ be any vector space, and let $E^{\prime}$ be a subspace of its algebraic dual which contains all linear forms bounded in the topology $W\left(E, E^{\prime}\right)$. Such an $E^{\prime}$ can be obtained by choosing a weak-star dense subspace $F$ of the algebraic dual and letting $E^{\prime}$ consist of all linear forms bounded in the topology $W(E, F)$. If $V$ is a neighborhood in the ordinary topology on $E^{\prime}$, then the space $E_{v}$ of all $x$ in $E$ such that $v(x)=\sup \{|(f, x)|: f$ in $V\}$ is finite is a normed linear space with $v$ as norm. A set is a neighborhood of the origin in the $G F$ topology defined by the $E_{v}$ if it is convex and circled and its intersection with each $E_{v}$ is a norm neighborhood in $E_{v}$. The next theorem shows that with the $G F$ topology defined by the $E_{v}, E$ is a symmetric and boundedly closed locally convex space.

\section{Theorem 1. The GF topology defined by the $E_{v}$ is the topology $T\left(E, E^{\prime}\right)$.}

Proof. Let $W$ be a neighborhood in $T\left(E, E^{\prime}\right)$. For each $V$, the unit sphere in $E_{v}$, being the polar of $V$, is bounded in $E$. For each $V$, therefore, a contraction of the unit sphere in $E_{v}$ is contained in $W$; and $W$ is a $G F$ neighborhood.

Let $W$ be a $G F$ neighborhood. Each bounded set in $E$ is contained in the unit sphere of some $E_{v}$ and therefore in a multiple of $W$. It follows that a linear form which is bounded on $W$ is bounded, which implies that $W^{\circ}$ is weak-star complete. $W^{\circ}$ is clearly weak-star bounded. From the equivalence of weak-star bounded and weak-star total bounded we deduce that $W^{\circ}$ is weak-star compact. In this way we see that $W^{\circ O}$ is a $T\left(E, E^{\prime}\right)$ neighborhood and we must show that $W$ itself is.

For any $x$ in $E$ we obtain from the Hahn-Banach theorem a linear form $f$ on $E$ satisfying $(f, x)=p(x)$ and $|(f, y)| \leqq p(y)$ for all $y$ in $E$, where $p$ is the semi-norm associated with $W$. The latter inequality implies that $f$ is bounded, and that it in fact lies in $W^{\circ}$. Thus if $x$ is in $W^{\circ}, p(x)=(f, x) \leqq 1$, so that $p$ is continuous $T\left(E, E^{\prime}\right)$, and $W$ is a $T\left(E, E^{\prime}\right)$ neighborhood.

We abstract the essential elements of the above $G F$ topology to obtain the general definition.

Let $E$ be a linear space, and let $E_{v}$ be a family of symmetric and boundedly closed locally convex spaces. For every $v$ let $T_{v}$ be a linear transformation of $E_{v}$ into $E$, and let the union of the sets $T_{v}\left(E_{v}\right)$ span $E$. The $G F$ topology on $E$ defined by $\left\{E_{v}, T_{v}\right\}$ is the strongest locally convex topology on $E$ making the mappings $T_{v}$ continuous, provided such a topology exists.

THEOREM 2. If E admits any locally convex topology making the mappings 
$T_{v}$ continuous, then the $G F$ topology on $E$ defined by $\left\{E_{v}, T_{v}\right\}$ exists.

We omit the obvious proof.

Suppose that a $G F$ topology exists on the space $E$, and denote it by $T^{*}$. If $W$ is a set in $T^{*}$, the continuity of the transformation $T_{v}$ implies that $T_{0}^{-1}(W)$ contains a neighborhood of the origin in $E_{v}$. It is also clear that any set $W$ having this property belongs to $T^{*}$. The attempt to define the $G F$ topology directly in terms of the neighborhoods identified above leads to a topology on $E$ which satisfies all of the axioms characterizing a locally convex space except the separation axiom. Accordingly it seems preferable to define the $G F$ space in the manner adopted. We give an example of a system $\left\{E, E_{v}, T_{v}\right\}$ where the $G F$ topology does not exist. Let $E$ be a two-dimensional vector space spanned by the vectors $i$ and $j$. Let $E_{i}$ and $E_{j}$ be infinite-dimensional Hilbert space. Let $f$ be a continuous linear form on $E_{i}$, and let $g$ be a discontinuous linear form on $E_{j}$. Define $T_{i}(x)=(f, x) i$ and $T_{j}(x)=(g, x) j$. If $S$ is any sphere in $E_{j}, T_{j}(S)$ contains the whole subspace spanned by the vector $j$. Hence there is no separated topology making $T_{j}$ continuous.

A locally convex space is called a $G F$ space if its topology is a $G F$ topology.

THEOREM 3. Every GF space is symmetric and boundedly closed. Every symmetric and boundedly closed space is a GF space determined by a family of normed linear spaces.

Proof. Suppose $f$ is a bounded linear form on $E$. The function $\left(f, T_{v}(x)\right)$ is a bounded linear form on $E_{v}$, since the continuous mapping $T_{v}$ carries bounded sets in $E_{v}$ into bounded sets in $E$. The hypothesis that $E_{v}$ is boundedly closed thus implies that $\left(f, T_{v}(x)\right)$ is continuous on $E_{v}$. If $V$ is the set of all $z$ in $E$ for which $|(f, z)| \leqq 1$, then $T_{v}^{-1}(V)$ is the set of all $x$ in $E_{v}$ for which $\left|\left(f, T_{v}(x)\right)\right| \leqq 1$, which, as we have seen, contains a neighborhood in $E_{v}$. Hence $V$ is a $G F$ neighborhood and $f$ is continuous.

The argument to show that $E$ is symmetric is similar. Let $B$ be a bounded set in $E^{\prime}$. The linear forms $\left(f, T_{v}(x)\right)$ for $f$ in $B$ form a bounded set in $E_{v}^{\prime}$, which by the symmetry of $E_{v}$ is contained in the polar of a neighborhood $U_{v}$ in $E_{v}$. Since $T_{v}^{-1}\left(B^{\circ}\right)$ contains $U_{v}, B^{\circ}$ is a neighborhood in the $G F$ topology of $E$.

That every symmetric and boundedly closed space is a $G F$ space determined by a family of normed linear spaces follows directly from Theorem 1 , and the discussion preceding it. These considerations, together with Theorem 3, give us:

THEOREM 4. There is at most one GF topology on E yielding a prescribed dual. In particular every $G F$ topology is of the type described at the beginning of this section.

The concept of a $G F$ topology is most fruitful when applied to the dual of a locally convex space. There are in fact two natural $G F$ topologies on the 
dual. One is obtained by taking as $E_{0}^{\prime}$ the normed linear space of all $f$ in $E^{\prime}$ such that $v(f)=\sup \{|(f, x)|: x$ in $V\}$ is finite, where $V$ is a neighborhood in $E$. The other is obtained in the same way, but where $V$ is a neighborhood in $E^{\prime \prime}$. In each case it is clear that the identity transformation $T_{v}$ from $E_{v}^{\prime}$ to $E^{\prime}$ is continuous with respect to the ordinary topology of $E^{\prime}$ so that the $G F$ topology exists. It follows from Theorem 1 that the latter, which is the weaker of the two, is the topology $T\left(E^{\prime}, E^{\prime *}\right), E^{\prime *}$ being the space of bounded linear forms on $E^{\prime}$. If $E$ is symmetric, the two $G F$ topologies coincide, and the interesting case occurs when they do coincide and coincide also with the ordinary topology of $E^{\prime}$. When this situation obtains, we say that $E$ has a $G F$ dual. If $E$ is not symmetric, the two $G F$ topologies may not coincide. An example is Banach space in its weak topology.

We obtain an interior mapping theorem for spaces with $G F$ duals. The first step is the following lemma, which also plays a critical role in several later theorems.

LEMMA. Let $A$ and $B$ be closed convex circled subsets of a locally convex space $E$. Then $(A \cap B)^{\circ}$ is the weak-star closure of the convex envelope of $A^{\circ}$ and $B^{\circ}$; if both $A$ and $B$ are compact, their convex envelope is compact; if either is compact, their sum $A+B$ is closed.

Proof. The first statement is proved by Dieudonné and Schwartz [7], the second assertion is elementary, while the last is a standard theorem.

THEOREM 5. Let $T$ be an isomorphism of $E$ into any locally convex space $F$ such that $T(E)$ is closed in $F$. If $E$ has a GF dual, then the adjoint $T^{*}$ is a homomorphism of $F^{\prime}$ onto $E^{\prime}$.

Proof. By definition a linear transformation $T$ of $E$ into $F$ is a homomorphism of $E$ into $F$ if it is continuous and interior between $E$ and $T(E)$; that is, continuous and such that to each neighborhood $V$ in $E$ corresponds a neighborhood $U$ in $F$ with $U \cap T(E) \subseteq T(V) . T$ is an isomorphism if it is a homomorphism and one to one. It is well known that the adjoint of a continuous linear transformation is a continuous linear transformation itself [7]. It remains to show that $T^{*}$ is onto and interior. That $T^{*}$ is onto follows from a theorem of Dieudonné [5]. On the other hand, it is as difficult to show that the theorem of Dieudonné is applicable as to give a direct proof. Let $f$ be arbitrary in $E^{\prime}$. The equation $(g, T x)=(f, x)$ defines $g$ as a linear form on $T(E)$. If $f$ is bounded on the neighborhood $V$ in $E$, then $g$ is bounded on $T(V)$ which is a neighborhood in $T(E)$, so that $g$ is continuous on $T(E)$. Let $h$ be a continuous extension of $g$ to all of $F$. Then for each $x$ in $E,\left(T^{*} h, x\right)=(g, T x)$ $=(f, x)$, and $T^{*} h=f$.

Finally, $T^{*}$ is interior. Let $V$ be an arbitrary neighborhood in $E$ and choose the neighborhood $U$ in $F$ so that $U \cap T(E) \subseteq T(V)$. As $U$ and $T(E)$ are both closed and $U^{\circ}$ is weak-star compact, it follows from the lemma 
that $T(V)^{\circ} \subseteq(U \cap T(E))^{\circ}=U^{\circ}+T(E)^{\circ}$. Recalling that $T^{*}$ is onto we see that $V^{\circ} \subseteq T^{*}\left((T V)^{\circ}\right)$. The collected inequalities and the fact that $T(E)^{\circ}$ is the null space of $T^{*}$ give $V^{\circ} \subseteq T^{*}\left(U^{\circ}\right)$. If $W$ is an arbitrary neighborhood in $F^{\prime}$, there is a number $\lambda$ such that $U^{\circ} \subseteq \lambda W$, so that $V^{\circ} \subseteq \lambda T^{*}(W)$. In other words, the image of an arbitrary neighborhood in $F^{\prime}$ is a $G F$ neighborhood in $E^{\prime}$.

Corollary. If $E$ is a closed subspace of the locally convex space $F$, and $E$ has a $G F$ dual, then $E^{\prime}=F^{\prime} / E^{\circ}$.

Proof. The identity transformation from $E$ to $F$ satisfies the conditions of the theorem. Since the null space of $T^{*}$ is $T(E)^{\circ}=E^{\circ}, T^{*}$ can be regarded as a transformation from $F^{\prime} / E^{\circ}$ to $E^{\prime}$. It is a direct consequence of the definition of the quotient topology that $T^{*}$ is an isomorphism.

REMARK. If $E$ is a closed subspace of the locally convex space $F$, if $F^{\prime}$ is boundedly closed, and if $E^{\prime}=F^{\prime} / E^{\circ}$, then $E^{\prime}$ is boundedly closed.

Proof. Every bounded linear form on $E^{\prime}$ has an obvious extension to a bounded linear form on $F^{\prime}$. The form on $E^{\prime}$ is bounded on the canonical image of the neighborhood on which the extension is bounded.

THEOREM 6. Every closed reflexive subspace of a reflexive space with a $G F$ dual has itself a GF dual.

Proof. Let $F$ be the space and $E$ the subspace. Since $E$ is reflexive, the neighborhoods in $E^{\prime}$ are the polars of weak-star compact sets in $E^{\prime \prime}=E$. Every reflexive space is obviously symmetric, so that, in view of Theorem 1, it is sufficient to show that $E^{\prime}$ is boundedly closed. Dieudonné and Schwartz show that whenever $F$ is reflexive and $E$ is a closed subspace of $F, E^{\prime}$ $=F^{\prime} / E^{\circ}[7]$. The remark above completes the proof.

THEOREM 7. If $H$ is a closed subspace of the symmetric space $E$, then $E / H$ is symmetric.

Proof. Let $T$ be the canonical mapping of $E$ on $E / H$. If $B$ is a bounded subset of $(E / H)^{\prime}$, then $T^{*}(B)$ is a bounded subset of $E^{\prime}$. When $E$ is symmetric, $T^{*}(B)$ is bounded on a neighborhood $V$, and $B$ is bounded on the neighborhood $T(V)$ in $E / H$.

If it is also true that $E / H$ has a $G F$ dual whenever $E$ has a $G F$ dual, then the interior mapping theorem is valid for homomorphisms $T$ as well as isomorphisms. We do not know if this is the case.

One might suspect that an analogous theorem holds for $H$ : that is, if $E$ is symmetric, then the closed subspace $H$ is symmetric. We have shown that every locally convex space is a subspace of a direct product of Banach spaces, and it is clear that a complete space is a closed subspace of the direct product. We show later that a product of Banach spaces is always symmetric; we 
know, however, that there exist complete nonsymmetric spaces. An example of one will be given later.

The succeeding sections are devoted to discussion of the $G F$ topology and the related concepts of symmetry and bounded closure in certain general types of locally convex space: direct products and their duals, continuous function spaces and their duals, and the duals of $F$ and $L F$ spaces. This section is concluded with a few particular examples.

1. Every metric or $L F$ space is a $G F$ space, for such spaces are symmetric and boundedly closed.

2. The space $L^{\omega}$ is a $G F$ space and it has a $G F$ dual. $L^{\omega}$ itself is a reflexive $F$ space, and therefore a $G F$ space. We show later that every reflexive $F$ space has a $G F$ dual. The dual of $L^{\omega}$, however, is especially interesting as an example of the general theory, so we discuss it in some detail here.

Let $E$ be the space $L^{\omega}$, consisting of those measurable functions on the closed interval $(0,1)$ which are $p$-summable for all $p>1$. $E$ can be regarded as the intersection of all the spaces $L^{p}$ for $p>1$. E is topologized by taking the $L^{p}$ norms as a family of semi-norms. It is easy to see that $E$ is an $F$ space in this topology.

Every $f$ in $E^{\prime}$ is bounded on a neighborhood in $E$, and hence can be regarded as an element of the dual of some $L^{p}$, which is an $L^{q}$. On the other hand it is clear that every element of an $L^{q}$ is in $E^{\prime}$; and $E^{\prime}$ is the union of the spaces $L^{q}$ for $q>1$. Let $T_{q}$ be the identity transformation from $L^{q}$ into $E^{\prime}$. The unit sphere in $L^{q}$, being the polar of a neighborhood in $E$, is bounded in $E^{\prime}$, so that the mapping $T_{q}$ is continuous, and the $G F$ topology defined by $\left\{L^{q}, T_{q}\right\}$ exists. This $G F$ topology is clearly the stronger of the two natural $G F$ topologies. At the same time $E$ is metric, hence symmetric, so that the two natural $G F$ topologies coincide, and we can speak of the $G F$ topology on $E^{\prime}$.

The dual of $E^{\prime}$ with respect to the $G F$ topology is the space of bounded linear forms on $E^{\prime}$. Every $G F$ continuous linear form is continuous on each $L^{q}$, hence is in each $L^{p}$, hence belongs to $E$. We conclude that $E^{\prime}$ with its ordinary topology is boundedly closed and that $E$ is semi-reflexive. It follows that $E^{\prime}$ is symmetric (introduction). Thus the ordinary topology is a $G F$ topology whose dual is the same as that of the $G F$ topology above. Because of the uniqueness of the $G F$ topologies, the two coincide.

3. The ring of entire functions is a $G F$ space and it has a $G F$ dual. The space $E$ of entire functions is again a reflexive $F$ space, and one which profitably can be examined directly. Its topology is defined by a family of semi-norms $p_{n}, p_{n}(x)$ being the upper bound over the circle of radius $n$ of $|x(z)|$. The fact that the neighborhood $p_{n}(x) \leqq 1$ is compact with respect to the metric $p_{n-1}(x)$ is used to show that the $G F$ and ordinary topologies of the dual coincide on bounded sets. The well known fact that a subspace in the dual of an $F$ space is weak-star closed if its intersection with each weak-star compact set is weak-star closed and the reflexivity of $E$ then imply that the $G F$ dual of 
$E^{\prime}$ is $E$ itself. The remainder of the argument parallels the last part of the argument for $L^{\omega}$.

4. The space $D^{\prime}$ of distributions is a $G F$ dual. $D^{\prime}$ is the dual of the reflexive $L F$ space of all functions defined on Euclidean $n$-space which are infinitely differentiable and vanish outside compact sets [15]. We rely on our general theory, which asserts that every $L F$ space which is reflexive has a $G F$ dual, rather than discuss $D^{\prime}$ directly; but we mention the example because of its current interest.

5. A purely algebraic situation, discussed in part by Mackey [13], is of some interest. Let $E$ be an arbitrary linear space. If $H$ is a Hamel basis for $E$, then $E$ can be regarded as the space of all complex-valued functions on $H$ which vanish outside finite sets. Let $E^{\prime}$ be the algebraic dual of $E$. It is easy to see that $E^{\prime}$ is the space of all complex-valued functions on $H$, and that the weak-star topology on $E^{\prime}$ is that of pointwise convergence on $H$. In other words, $E^{\prime}$ is the direct product, with $H$ as index set, of complex planes. In the next section we show that a bounded set in the dual of a direct product contains only a finite number of nonzero components. Thus every ordinary neighborhood in $E^{\prime}$, as the polar of a bounded set in $E$, is a weak-star neighborhood, and the ordinary and weak-star topologies on $E^{\prime}$ coincide.

With respect to the topology $T\left(E, E^{\prime}\right)$ the mappings from the finitedimensional subspaces of $E$ to $E$ are continuous so that the $G F$ topology defined by the finite-dimensional subspaces, which are of course Euclidean, exists and is stronger than $T\left(E, E^{\prime}\right)$. The $G F$ topology must actually be the same as $T\left(E, E^{\prime}\right)$ because its dual can be no larger than $E^{\prime}$, which is the algebraic dual.

2. Direct products. In this section we examine the direct product of an arbitrary family of locally convex spaces. Let $A$ be an index set, $t$ the generic point of $A, E_{t}$ a locally convex space, and $E(A)$ the direct product of the $E_{t}$. $E(A)$ is composed of functions $x(t)=x_{t}$ defined on $A$ for which $x_{t} \in E_{t}$. A neighborhood of the origin in $E(A)$ is determined by a finite set $F$ of indices and neighborhoods $V_{t}$ in $E_{t}$ for $t$ in $F$. It consists of all $x(t)$ for which $t$ in $F$ implies $x_{t}$ in $V_{t}$.

We use consistently the following notation: $E=E(A), N$ is the subspace of $E$ consisting of functions with only a finite number of nonzero components, and $N^{\prime}$ is the corresponding subspace of the direct product of the spaces $E_{t}^{\prime}$. By the identity mapping of $E_{t}$ into $E$ we mean the transformation $I_{t}$ which carries $x_{t}$ in $E_{t}$ into that $x$ in $E$ which is $x_{t}$ at the index $t$ and 0 elsewhere. We call its adjoint, which carries $E^{\prime}$ onto $E_{t}^{\prime}$, the projection of $E^{\prime}$ on $E_{t}^{\prime}$. Since the identity is continuous, projection is both strong and weak-star continuous. Using the identity mapping, we can regard $E_{t}$ as a subspace of $E$.

We proceed to identify the dual $E^{\prime}$.

THEOREM 1. $E^{\prime}$ and $N^{\prime}$ are algebraically isomorphic. 
Proof. If $f(t)$ is in $N^{\prime}$, we write $(f(t), x)=\sum\left(f_{t}, x_{t}\right)$ to obtain a one to one linear mapping of $N^{\prime}$ into $E^{\prime}$. To show that the mapping is onto, we note that every $f$ in $E^{\prime}$ is bounded on a neighborhood, so that only a finite number of its projections are different from 0 , and $f(t)=I_{t}^{*} f$ belongs to $N^{\prime}$. Clearly the image of $f(t)$ and $f$ coincide on $N$, which is dense in $E$.

Corollary. The topology $W\left(E, E^{\prime}\right)$ is the product of the topologies $W\left(E_{t}, E_{t}^{\prime}\right)$.

As in the case of $E$, we may now speak of the identity transformation of $E_{t}^{\prime}$ into $E^{\prime}$. Since the image of $E_{t}^{\prime}$ consists of linear forms which vanish on every $E_{t}$ save one, it is clear that the identity mapping is continuous: a net in $E_{t}^{\prime}$ converging uniformly on bounded sets in $E_{t}$ certainly has for an image a net converging uniformly on bounded sets in $E$. Hence $T^{\prime}$, the ordinary topology of $E^{\prime}$, is stronger than the product of the topologies $T_{t}^{\prime}$. The identity is also weak-star continuous, so that its adjoint projects $E$ onto $E_{t}$. We note that a set in $E$ is bounded if and only if each of its projections is bounded.

THEOREM 2. Every bounded set in $E^{\prime}$ is contained in the direct product of finitely many $E_{t}^{\prime}$.

Proof. Let $B$ be a bounded set in $E^{\prime}$, and let $p(x)=\sup \{|(f, x)|: f$ in $B\}$; by hypothesis $p(x)$ is bounded on bounded sets in $E$. If the theorem is not true, there exist sequences $t_{n}$ in $A$ and $x_{n}$ in $E_{t_{n}}$ for which $p\left(I x_{n}\right)=n$. However, such a sequence $I x_{n}$ is bounded.

Corollary. The topology $T\left(E, E^{\prime}\right)$ is the product of the topologies $T\left(E_{t}, E_{t}^{\prime}\right)$.

Proof. Theorem 1 implies that if each factor is provided with the relatively strong topology, $E$ still has the dual $E^{\prime}=N^{\prime}$, whence $T\left(E, E^{\prime}\right)$ is stronger than the product of the $T\left(E_{t}, E_{t}^{\prime}\right)$. Now if $C$ is a convex circled weak-star compact set in $E^{\prime}$, the projection $C_{t}$ of $C$ on $E_{t}^{\prime}$ is $W\left(E_{t}^{\prime}, E_{t}\right)$ compact by virtue of the weak-star continuity of projection. Moreover, $C_{t}$ is 0 for all but finitely many $t$ in $A$, since $C$ is bounded and Theorem 2 applicable. It follows that $C$ is uniformly bounded on the product neighborhood determined by the nontrivial $C_{t}$, and the product of $T\left(E_{t}, E_{t}^{\prime}\right)$ is stronger than $T\left(E, E^{\prime}\right)$.

THEOREM 3. $E$ is symmetric if and only if each $E_{t}$ is symmetric.

Proof. If each $E_{t}$ is symmetric, and $B$ is bounded in $E^{\prime}$, the projection $B_{t}$ of $B$ on $E_{t}^{\prime}$ is bounded, since projection is continuous. $B_{t}$ is 0 except for a finite number of $t$ because of Theorem 2. The direct product of $\left(B_{t}\right)^{\circ}$ is a neighborhood in $E$ upon which $B$ is uniformly bounded, i.e., $E$ is symmetric.

If the factor $E_{t_{0}}$ is not symmetric, there exists a bounded set $B_{0}$ in $E_{t_{0}}^{\prime}$ which is not uniformly bounded on a neighborhood in $E_{t_{0}}$. The identity 
mapping of $E_{\ell_{0}}^{\prime}$ into $E^{\prime}$ provides a bounded set in $E^{\prime}$ which is bounded on no neighborhood in $E$, whence $E$ is not symmetric.

Theorem 4. If each $E_{t}$ has a GF dual, then E has a GF dual.

Proof. Let $W$ be a neighborhood in the stronger of the two natural $G F$ topologies. Then $W \cap E_{t}^{\prime}$ is a $G F$ neighborhood in $E_{t}^{\prime}$, and by hypothesis there is a bounded set $B_{t}$ in $E_{t}$ such that $W \cap E_{t}^{\prime}$ contains $B_{t}^{\circ} \cap E_{t}^{\prime}$. Let $B$ be the direct product of the sets $B_{t} . B$ is bounded and $B^{\circ} \cap E_{t}^{\prime}=B_{t}{ }^{\circ} \cap E_{t}^{\prime} \subseteq W$.

Let $F$ be any set of $n$ points in $A$, and let $E_{F}{ }^{\prime}$ be the direct product of the spaces $E_{t}^{\prime}$ for $t$ in $F$. Let $f$ be an arbitrary element of $B^{\circ} \cap E_{F}^{\prime}$ : The projection $f_{t}$ of $f$ on $E_{t}^{\prime}$ is in $B_{t}^{\circ}$. Set $\alpha_{t}=\sup \left\{\left(f_{t}, x_{t}\right): x_{t}\right.$ in $\left.B_{t}\right\}$. Since we assume $B$ circled, for $\epsilon>0$ there exists $x_{t}$ in $B_{t}$ with $\left(f_{t}, x_{t}\right)>\alpha_{t}-\epsilon / n$. The $x$ in $E$ which is $x_{t}$ if $t$ is in $F$ and is 0 otherwise is in $B$ so that $1 \geqq(f, x)=\sum\left(f_{t}, x_{t}\right) \geqq \sum \alpha_{t}$ $-\epsilon$; it follows that $\sum \alpha_{t} \leqq 1$. Writing $f=\sum \alpha_{t}\left(f_{t} / \alpha_{t}\right)$ and noting that $f_{t} / \alpha_{t}$ is in $W$, we conclude that $f$ is in $W$. Since every $f$ in $B^{\circ}$ is in some $B^{\circ} \cap E_{F}^{\prime}$, we have $B^{\circ} \subseteq W$.

This implies that the ordinary topology, which is weaker than the weak $G F$ topology, is also stronger than the strong $G F$ topology.

TheOREM 5. Every bounded linear form on $E$ is continuous on $N$.

Proof. If this were not so, there would exist sequences $t_{n}$ in $A$ and $x_{n}$ in $E_{t_{n}}$ such that $\left(f, I x_{n}\right)=n$. The sequence $I x_{n}$, however, is bounded.

CoRollary. $E$ is boundedly closed if and only if every bounded linear form on $E$ is uniquely determined by its behavior on $N$.

Proof. The restriction of $f$ to $N$ is continuous there; since $N$ is dense in $E$, $f$ has a continuous extension over $E$.

CoRollary. $E$ is boundedly closed if and only if $N$ is dense in $E$ in the weak topology determined on $E$ by the space of bounded linear forms.

Corollary. $E$ is not boundedly closed if and only if there exists a nonzero bounded linear form on $E$ which vanishes on $N$.

We suppose now that the factor spaces $E_{t}$ are metric spaces and consider the question of the bounded closure of $E$. Some of the results which follow have been obtained in a less general context by Mackey [11] and Ulam [17]; these authors, however, employ arguments essentially different from ours.

We suppose that $f$ is some fixed nontrivial bounded linear form on $E=E(A)$, and that $L$ is the class of subsets $K$ of $A$ such that $x(t)=0$ on $K$ implies $(f, x)=0$. It is convenient to present our argument in a sequence of lemmas.

Lemma 1. If $A$ is the union of an ascending sequence of sets $A_{n}$, there exists $N$ such that $n \geqq N$ implies $A_{n}$ is in $L$. 
Proof. If no $A_{n}$ is in $L$, there exists, for every $n$, an $x_{n}$ in $E$ which vanishes on $A_{n}$ and for which $\left(f, x_{n}\right)=n$. This cannot happen, however, since any such sequence is bounded. It follows that some $A_{n}$ is in $L$, and obviously any set containing a set in $L$ is also in $L$.

Lemma 2. There exist neighborhoods $V_{t}$ in $E_{t}$ such that $f$ is bounded on $V$, the direct product of the $V_{t}$.

Proof. Write the neighborhood system in $E_{t}$ as $V_{t n}$, and for each $n$ let $V_{n}$ be the direct product of the sets $V_{t n}$. If $f$ is unbounded on $V_{n}$, there exists $x_{n}$ in $V_{n}$ with $\left(f, x_{n}\right)=n$. The sequence $x_{n}$ converges to 0 , hence is a bounded set, and $\left(f, x_{n}\right)=n$ is impossible.

Lemma 3. $f$ is sequentially continuous.

Proof. We suppose that there is a sequence $x_{n}$ converging to 0 in $E$ for which $\left|\left(f, x_{n}\right)\right|>1$ and deduce a contradiction. Choose the neighborhoods $V_{t}$ in $E_{t}$ so that $|(f, x)| \leqq 1$ for all $x$ in $V$, their direct product. Let $A_{n}$ be the set of all $t$ in $A$ for which $m \geqq n$ implies $x_{m}(t) \in V_{t} . A$ is the union of the ascending sequence $A_{n}$, and by Lemma 1 there exists $N$ such that $A_{N}$ is in $L$. Let $y_{n}$ be the element of $E$ which coincides with $x_{n}$ on $A_{N}$ and vanishes elsewhere; clearly $\left(f, y_{n}\right)=\left(f, x_{n}\right)$. For every $n \geqq N, y_{n}$ is in $V$, whence $\left|\left(f, x_{n}\right)\right|$ $=\left|\left(f, y_{n}\right)\right| \leqq 1$.

\section{Lemma 4. The family $L$ is closed under finite or countable set intersection.}

Proof. If $B$ and $D$ are sets in $L$, and $x$ in $E$ vanishes on $B \cap D$, let $y$ be that element of $E$ which vanishes on $B$ and coincides with $x$ elsewhere. Writing $x=y+x-y$. we see that $(f, x)=(f, y)+(f, x-y)=0$, since $y$ vanishes on $B$ and $x-y$ on $D$. It follows that $L$ is closed under finite intersection.

If the sets $D_{n}$ form a descending sequence of sets in $L$ and $x$ in $E$ vanishes on $\cap D_{n}$, let $y_{n}$ be that element of $E$ which vanishes on $D_{n}$ and coincides with $x$ elsewhere. Then $y_{n}$ converges to $x$ and $\left(f, y_{n}\right)=0$ for all $n$; the sequential continuity of $f$ implies that $(f, x)=0$.

Lemma 5. Let $Q$ be the class of all subsets of $A$ which intersect every set in $L$. $Q$ contains at most finitely many disjoint sets.

Proof. If $B_{n}$ is a sequence of disjoint sets in $Q$, let $A_{0}$ denote the complement of their union, and let $A_{n}=\cup_{i=1}^{n}\left(A_{0} \cup B_{i}\right)$. The $A_{n}$ form an ascending sequence of sets whose union is $A$. By Lemma 1 there exists $N$ such that $A_{N}$ is in $L$. If $n>N, B_{n}$ fails to intersect $A_{N}$, hence is not in $Q$.

Lemma 6. If $M$ is a set in $Q$ which is the union of finitely many disjoint sets $M_{i}$, then at least one $M_{i}$ is in $Q$.

Proof. If not, then for every $M_{i}$ there is a set $A_{i}$ in $L$ such that $M_{i} \cap A_{i}$ is empty. $A_{0}=\cap A_{i}$ is in $L$ and $M \cap A_{0}$ is empty, so that $M$ is not in $Q$. 
Leмma 7. Let $M_{i}$ be a maximal collection of disjoint sets in $Q$ and suppose that $N_{k}$ is another such collection. There exists a maximal collection $M_{i}^{*}$ which is a refinement of both $M_{i}$ and $N_{k}$.

Proof. Consider the family of sets $M_{1} \cap N_{k}$. It is easy to see that one and only one of these sets is in $Q$. Call it $M_{1}^{*}$. Form similarly the other sets $M_{i}^{*}$. Evidently each $M_{i}^{*}$ is contained in the intersection of an $M_{i}$ and an $N_{k}$, and the collection $M_{i}^{*}$ is a maximal collection of disjoint sets in $Q$.

We introduce a set of measures associated with a maximal family of disjoint sets in $Q$. If $S$ is a subset of $A$, write $m_{i}(S)=1$ if $M_{i} \cap S$ is in $Q, m_{i}(S)=0$ otherwise.

LeMmA 8. Each $m_{i}$ is a countably additive measure on $A$ for which every subset of $A$ is measurable.

Proof. It is clear that we have only to show the countable additivity. Let $S$ be the union of a sequence of disjoint sets $S_{n}$, and let $m$ be one of the $m_{i}$. At most one of the $S_{n}$ has positive measure, since $m\left(S_{n}\right)=m\left(S_{k}\right)=1 \mathrm{im}$ plies that $M_{i} \cap S_{n}$ and $M_{i} \cap S_{k}$ are disjoint sets in $Q$, contrary to the maximality of the family $M_{i}$. If one $S_{n}$, say $S_{0}$, does have positive measure, clearly $m(S)=m\left(S_{0}\right)=1$. If no $S_{n}$ has positive measure, then there exists for each $n$ a set $A_{n}$ in $L$ such that $M_{i} \cap S_{n} \cap A_{n}$ is empty. Because of Lemma $4, A_{0}=\cap A_{n}$ is in $L$, and as $M_{i} \cap S_{n} \cap A_{0}$ is empty for all $n, M_{i} \cap S \cap A_{0}$ is empty. Thus $M_{i} \cap S$ fails to intersect a set in $L$ and cannot lie in $Q$ so that $m(S)=\sum m\left(S_{n}\right)$ $=0$.

\section{LEMMA 9. The measures $m_{i}$ depend only on the linear form $f$.}

Proof. The set families $L$ and $Q$ depend only on $f$; we have to show that the $m_{i}$ do not depend on the particular choice of the maximal family $M_{i}$. Let $N_{k}$ be another family of disjoint sets in $Q$ which is also maximal, and let $M_{i}^{*}$ be the refinement of $M_{i}$ and $N_{k}$ provided by Lemma 7 . Now $m_{i}(S)=1$ if and only if $M_{i} \cap S$ is in $Q$, which happens if and only if $M_{i}^{*} \cap S$ is in $Q$, i.e., $m_{i}(S)=1$ if and only if $m_{i}^{*}(S)=1$. Similarly, after the subscripts have been suitably arranged, $n_{k}(S)=1$ if and only if $m_{k}^{*}(S)=1$. The measures $m_{i}$ and $n_{k}$ then coincide, except possibly for order.

Lemma 10. If $x$ is in $E$ and $D$ is a subset of $A$ outside of which $x(t)=0$, then $m_{i}(D)=0$ for all $i$ implies $(f, x)=0$.

Proof. For each $i$ there exists $A_{i}$ in $L$ such that $M_{i} \cap D \cap A_{i}$ is empty. If $A_{0}$ is the intersection of the $A_{i}$, then $A_{0}$ is in $L$ and $M_{i} \cap D \cap A_{0}$ is empty for all $i$. Since the $M_{i}$ are maximal, $D \cap A_{0}$ cannot be in $Q$ and there exists $A^{*}$ in $L$ such that $D \cap A_{0} \cap A^{*}$ is empty. $A_{0} \cap A^{*}$ is a set in $L$ lying in the complement of $D$; hence $x(t)=0$ on a set in $L$, and $(f, x)=0$.

We sum up our results in the following theorem. 
THEOREM 6. If $f$ is a bounded linear form on $E$, the direct product of metric spaces, $f$ is sequentially continuous; there is associated with $f$ a finite set of atomic measures $m_{i}$ on $A$ such that $m_{i}(A)=1$, every subset of $A$ is $m_{i}$-measurable, $m_{i}$ is countably additive, and if $x(t)$ in $E$ vanishes on a set of positive $m_{i}$ measure for all $i$, then $(f, x)=0$.

It is easy to see that if the bounded linear form $f$ is continuous, then to each $i$ there corresponds a point $t_{i}$ of $A$, and this collection of points forms the unique maximal collection of disjoint sets in $Q$. If, however, $f$ is not continuous, then at least one of the associated measures vanishes on finite sets. The problem of the existence of such a measure on an arbitrary set $A$ has been considered by Ulam [17]. Let us agree to call a measure $m$ on $A$ a $U$ measure if $m$ is countably additive, $m(A)=1, m$ is atomic and vanishes on finite sets, and every subset of $A$ is $m$-measurable. It is clear that if the set $A$ admits no $U$-measure, then $E(A)$ is boundedly closed. It is easy to establish the converse. If $m$ is a $U$-measure on $A$, then every complex-valued function on $A$ is measurable and indeed integrable. If for every $t$ in $A$ we choose a nonzero $f_{t}$ in $E_{t}^{\prime}$, and define $(g, x)=\int_{A}\left(f_{t}, x_{t}\right) d m$, it is clear that $g$ is a bounded discontinuous linear form on $E(A)$. Thus we have the following:

Corollary. $E(A)$, the direct product of metric spaces, is boundedly closed if and only if $A$ admits no $U$-measure.

We are now in a position to drop the hypothesis that the factor spaces $E_{t}$ are metric spaces and to obtain the following theorem.

THEOREM 7. $E(A)$, the direct product of boundedly closed locally convex spaces, is boundedly closed if and only if $A$ admits no $U$-measure.

Proof. That the existence of a $U$-measure on $A$ implies that $E(A)$ is not boundedly closed has been established in the proof of the corollary above, where no use was made of the hypothesis that the spaces $E_{t}$ were metric.

If $E(A)$ is not boundedly closed, by the corollary of Theorem 5 there exists a bounded linear form $f$ vanishing on $N$. Choose $x$ in $E(A)$ such that $(f, x) \neq 0$, and for every complex-valued function $y(t)$ on $A$ write $(g, y)$ $=\left(f, \sum y(t) x_{t}\right)$ where $x_{t}$ is the projection of $x$ on $E_{t} . g$ is a bounded discontinuous linear form on the direct product of complex planes; hence by the corollary above, there exists a $U$-measure on $A$.

From Theorem 7 it follows that the bounded closure of $E(A)$ depends only on the cardinality of the set $A$, and in no way on the character of the locally convex spaces $E_{t}$.

Suppose that $\boldsymbol{\aleph}^{\prime}$ is the smallest cardinal number for which there exists a set $A$ of that cardinality which admits a $U$-measure. Ulam [17] has shown: (1) $\boldsymbol{N}^{\prime}$ is a strongly inaccessible cardinal, and (2) $\boldsymbol{N}<\boldsymbol{N}^{\prime}$ implies $2 \boldsymbol{N}<\boldsymbol{N}^{\prime}$, whence $\boldsymbol{\aleph}^{\prime}$ is at least as great as the cardinal $\sum_{n=0}^{\infty} C(n)$, where $C(0)=\boldsymbol{\aleph}_{0}$ and $C(n)=2^{C(n-1)}$. 
3. Continuous function spaces. In this section, we investigate the status of bounded closure, symmetry, and $G F$ topologies in continuous function spaces and their duals. We use the following notation.

$S$ is any locally compact Hausdorff space. $E=C(S)$ is the vector space of all complex-valued continuous functions on $S$. The topology on $E$ is called the compact-open topology, and is that obtained by taking as neighborhoods of the origin all sets of the form: $V(C, \epsilon)=$ all $x(t)$ in $E$ which are at most $\epsilon$ in absolute value on $C$, where $C$ is a compact subset of $S$ and $\epsilon$ is positive. Convergence in $E$ is uniform convergence on compact subsets of $S . H(C)$ is the subspace of $E$ consisting of all functions which vanish on $C$; or, equivalently, the null space of the semi-norm associated with the neighborhood $V(C, \boldsymbol{\epsilon})$.

Let $V=V(C, \epsilon)$ be a neighborhood in $E$ and let $v(x)$ be the associated semi-norm. $E_{v}=E / H(C)$ with $v(x)$ as norm is the usual Banach space of continuous functions on the compact Hausdorff space $C$. Its dual $\left(E_{v}\right)^{\prime}$ is the space $\left(E^{\prime}\right)_{v}$ appearing in the definition of the stronger of the two natural $G F$ topologies on $E^{\prime}$. Each $f$ in $E^{\prime}$ lies in some $\left(E^{\prime}\right)_{v}=\left(E_{v}\right)^{\prime}$ and is therefore a measure on the set $C$; or, if we wish, a measure on $S$ which vanishes outside $C$. Thus $E^{\prime}$ can be described as the space of all Borel measures on $S$ which vanish outside a compact set.

It is easy to see that the bounded sets in $E^{\prime}$ which are bounded on a neighborhood consist of measures all of which vanish outside a common compact set. For some Hausdorff spaces $S$ there exist bounded subsets of $E^{\prime}$ without this property, as we shall show by an example, so there exist spaces $E=C(S)$ which are not symmetric. On the other hand, if $S$ is a countable union of compact sets, $E$ is metric and surely symmetric. In addition, we can state the following theorem.

TheOREM 1. If $S$ is paracompact, $E$ is symmetric.

Proof. By a result of Dieudonné, the paracompactness of $S$ implies that $E$ is a direct product of metric spaces, hence symmetric. We prefer, however, to give our own proof.

The paracompactness and local compactness insure the existence of a covering $\left(O_{\alpha}\right)$ of $S$ with the properties: (a) $O_{\alpha}$ is open and has compact closure and (b) no compact set intersects more than a finite number of the $O_{\alpha}$.

Let $B$ be a bounded subset of $E^{\prime}$, and set $p(x)=\sup \{|(f, x)|: f$ in $B\}$. There is a finite set $F$ of indices such that $p(x)=0$ for every $x$ whose support is contained in an $O_{\alpha}$ with $\alpha$ not in $F$. For in the contrary case we can find sequences $\alpha_{n}$ and $x_{n}$, with $x_{n}$ vanishing outside $O_{\alpha_{n}}$ and $p\left(x_{n}\right)=n$. Because of (b) above the sequence $x_{n}$ is bounded uniformly on each compact set, hence bounded in $E$, and $p\left(x_{n}\right)=n$ is impossible. Let $C$ be the closure of $\cup_{\alpha \in F} O_{\alpha}$.

Let $a_{\alpha}(t)$ be a partition of unity for the covering $O_{\alpha}$ [15]. The $a_{\alpha}(t)$ have the properties: $a_{\alpha}(t) \geqq 0 ; a_{\alpha}(t)=0$ outside $O_{\alpha}$; and for all $t, \sum a_{\alpha}(t)=1$. Each $x$ in $N$, the space of functions vanishing outside a compact set, can be written 
as a finite sum $x=\sum a_{\alpha} x$. If $x$ vanishes on $C$, each term in the sum has support contained in an $O_{\alpha}$ with $\alpha$ not in $F$ and $p(x) \leqq \sum p\left(a_{\alpha} x\right)=0$. Thus $p(x)$ vanishes on $H(C) \cap N, p(x)$, as a sup of continuous functions, is lower semicontinuous, and $H(C) \cap N$ is dense in $H(C)$, so that $p(x)$ vanishes on $H(C)$.

Let $D$ be the bounded set in $E$ of functions at most 1 in absolute value. $p(x)$ is clearly bounded on $H(C)+D$, which, as we shall see, contains a neighborhood.

Let $O$ be an open set with compact closure containing $C$, and let $e(t)$ be a function in $E$ which vanishes on $C$, equals 1 on the complement of $O$, and is between 0 and 1 elsewhere. Any $x$ in $V(\bar{O}, 1)$ can be written as $x(t)=e(t) x(t)$ $+(1-e(t)) x(t)$, and evidently $e(t) x(t)$ is in $H(C)$ while the other term is in $D$. Thus $V(\bar{O}, 1)$ is contained in $H(C)+D$.

THEOREM 2. If $E$ is symmetric, then the ordinary topology and the natural $G F$ topology coincide on bounded subsets of $E^{\prime}$.

Proof. Because $T^{\prime}$ is weaker than the $G F$ topology, it is sufficient to show that every bounded net $f_{\alpha}$ which converges to 0 for $T^{\prime}$ also converges to 0 for the $G F$ topology. The $f_{\alpha}$, being bounded, lie in the polar of a neighborhood $V(C, \boldsymbol{\epsilon})$. The $f_{\alpha}$ then vanish on $H(C)$. Since they converge to 0 uniformly on the set $D$ of functions at most 1 in absolute value, they converge to 0 uniformly on $H(C)+D$, which, as we have seen in the last theorem, contains a neighborhood $U$. The $f_{\alpha}$ thus converge to 0 in $E_{u}^{\prime}$, a fortiori in the $G F$ topology.

COROLlary. If $E$ is symmetric, then every bounded linear form on $E^{\prime}$ is continuous on bounded sets. In particular, every bounded linear form is sequentially continuous.

Proof. By the theorem, every bounded convergent net is $G F$ convergent. At the same time, every bounded linear form is $G F$ continuous.

THEOREM 3. If $S$ is a countable union of compact sets, then $E$ is symmetric and has a GF dual.

Proof. As we have observed, the symmetry follows from the fact that $E$ is metric. In view of Theorem 2 it will complete the proof to show that the dual of a metric space does not admit a locally convex topology which is properly stronger than the ordinary topology and which coincides with the latter on bounded sets. A. Grothendieck has announced this result without proof [9]. We therefore provide our own.

THEOREM 4. Let $E$ be a metric space with a descending sequence of neighborhoods $V_{n}$. If $W$ is a convex circled subset of $E^{\prime}$ such that $W \cap V_{n}^{\circ}$ is a neighborhood in $V_{n}^{\circ}$ for each $n$, then $W$ is a neighborhood in $E^{\prime}$.

Proof. By hypothesis we can find for each $n$ a bounded set $A_{n}$ in $E$ such that $A_{n}^{\circ} \cap V_{n}^{\circ} \subseteq W$. Let $a_{n k}$, for $k \geqq n$, be a number such that $A_{n} \subseteq a_{n k} V_{k}$, and 
let $c_{k}$ be a number such that $\left(c_{k} V_{k}\right)^{\circ} \subseteq W$. Setting $a_{k}=\max \left(a_{n k}, c_{k}, 1: n \leqq k\right)$, define $B=\bigcap_{k=1}^{\infty} a_{k} V_{k}, B_{n}=\bigcap_{k=n}^{\infty} a_{k} V_{k}$, and $C_{n}=\bigcap_{k=1}^{n-1} a_{k} V_{k}$. If $k \geqq n$, then $a_{k} \geqq a_{n k}$, and $a_{k} V_{k} \supseteq a_{n k} V_{k} \supseteq A_{n}$, so that $A_{n} \subseteq B_{n}$. Therefore $B_{n}^{\circ} \subseteq A_{n}^{\circ}$, and $B_{n}^{\circ} \cap V_{n}^{\circ} \subseteq A_{n}^{\circ}$ $\cap V_{n}^{\circ} \subseteq W$. By the lemma of the first section, $C_{n}^{\circ} \subseteq W$. Again by that lemma $B^{\circ} \cap V_{n}^{\circ}=\left(B_{n} \cap C_{n}\right)^{\circ} \cap V_{n}^{\circ} \subseteq\left(B_{n}^{\circ}+C_{n}^{\circ}\right) \cap V_{n}^{\circ}$, and we show that the last set is a subset of $4 W$. Every element of the last set is expressible in the form $f+g=h$, where $f$ is in $B_{n}^{\circ}, g$ is in $C_{n}^{\circ}$, and $h$ is in $V_{n}^{\circ}$. Since $C_{n}$ contains $V_{n}$, $C_{n}^{\circ}$ is contained in $V_{n}^{\circ}$, and $f=h-g$ is in $2 V_{n}^{\circ}$. Thus $f$ is in $2\left(V_{n}^{\circ} \cap B_{n}^{\circ}\right) \subseteq 2 W$, and $f+g$ is in $2 W+C_{n}^{\circ} \subseteq 4 W$. Since, now, $B^{\circ} \cap V_{n}^{\circ} \subseteq 4 W$ for every $n, B^{\circ} \subseteq 4 W$, or $(4 B)^{\circ} \subseteq W$.

We give the promised example of a continuous function space which is not symmetric.

THEOREM 5. If $S$ is sequentially compact but not compact, then $E$ is neither symmetric nor boundedly closed.

Proof. Each bounded subset $B$ of $E$ consists of functions bounded uniformly on $S$. If this were not the case, $B$ would fail to be bounded uniformly on some convergent sequence of points in $S$, which is impossible, since $B$ must be bounded uniformly on each compact set. In particular, each $x(t)$ in $E$ is bounded. We conclude immediately that the set of all point functionals (linear forms $f$ defined by a point $t_{0}$ in $S$ and the equation $(f, x)=x\left(t_{0}\right)$ ) is a bounded set in $E^{\prime}$. It is not bounded on a neighborhood, and $E$ is not symmetric.

Suppose for the moment that $E$ is the space of real rather than complex functions. It follows from the first paragraph that every non-negative linear form on $E$ is bounded, and that $\|x\|=\sup \{|x(t)|: t$ in $S\}$ is a well defined norm on $E$. A linear form which is continuous with respect to this norm is a difference of non-negative linear forms [3] and therefore is bounded.

The subspace $N$ of functions which vanish outside a compact set is not norm dense in $E$, so there exists a nonzero norm continuous linear form $f$ which vanishes on $N . f$ is bounded, but cannot be continuous since $N$ is dense in $E$.

To extend $f$ to the space of complex functions write the complex function $z(t)$ as $z(t)=x(t)+i y(t)$ where $x(t)$ and $y(t)$ are real. Then define $(f, z)=(f, x)$ $+i(f, y)$.

The representation theorems given in the introduction, Theorem 5, and the results of the preceding section can be used to show that closed subspaces do not inherit the properties of symmetry and bounded closure. A standard example of a Hausdorff space which is sequentially compact but not compact is obtained from the ordinal numbers. Let $c$ be the first uncountable cardinal and let $S$ be the first ordinal with cardinal number $c$ (equivalently, the set of all ordinals with cardinal number less than $c$ ). The neighborhoods of a point in $S$ are the intervals containing the point. Each interval $0 \leqq t \leqq t^{*}$ 
is compact and every compact set is contained in such an interval, so that the semi-norms defined by these intervals describe the topology of $C(S)$. Since there are $c$ intervals, $E$ is a subspace of a direct product of $c$ Banach spaces, and the latter, after $\$ 2$, is symmetric and boundedly closed. Thus $C(S)$ is neither symmetric nor boundedly closed, although it is a closed subspace of a space having both of those properties.

Even the relatively strong topology on $E=C(S)$ is not symmetric. We have shown that the bounded sets in $E$ are the norm bounded sets, which means that $E^{\prime}$ and $E^{\prime \prime}$ are both normed linear spaces. The relatively strong topology on $E$, however, is not metric, since $E$ is not boundedly closed.

Another example, especially interesting because it is semi-reflexive, of a space which is not symmetric in its relatively strong topology is the space $E=F^{\prime}$ with the topology $T\left(F^{\prime}, F\right)$, where $F$ is a nonreflexive Banach space. $E^{\prime}$ is $F$ in its norm topology and $E^{\prime \prime}$ is $F^{\prime}$ in its norm topology. As $F$ is not reflexive, the norm topology on $F^{\prime}$ is properly stronger than $T\left(F^{\prime}, F\right)$.

4. Metric and $L F$ spaces. We have observed that every metric space is symmetric, so that there is only one natural $G F$ topology on its dual. We show now that a metric space has a $G F$ dual whenever it has a symmetric dual. In particular every reflexive metric space has a $G F$ dual. We show also that every reflexive $L F$ space has a $G F$ dual.

Throughout this section, except in the last theorem, $E$ denotes a locally convex metric space. $E^{\prime *}$ denotes the space of bounded linear forms on $E^{\prime}$. The sets $B^{\circ}$ for $B$ a bounded set in $E^{\prime}$ form a neighborhood system in $E^{\prime *}$, as well as in $E^{\prime \prime}$, and topological terms refer to this topology unless some other is specifically mentioned.

Theorems 1 and 3 are generalizations of theorems announced without proof by A. Grothendieck [9].

THEOREM 1. Each bounded subset $B$ of $E^{\prime *}$ is contained in the weak-star closure (in $E^{* *}$ ) of a bounded subset $C$ of $E^{\prime \prime}$.

Proof. Let $V_{n}$ be the descending sequence of neighborhoods in $E$, and set $a_{n}=\sup \left\{|(z, f)|: f\right.$ in $V_{n}^{\circ}, z$ in $\left.B\right\}$. The convex envelope $W$ of the sets $1 / a_{n} V_{n}^{\circ}$ is a $G F$ neighborhood, so the set $W^{\circ}$ is bounded in $E^{\prime *}$, and $C=W^{\circ} \cap E^{\prime \prime}$ is bounded in $E^{\prime \prime}$.

First we show that each $z$ in $B$ is in the weak-star closure of $\bigcap_{k=1}^{n} a_{k} V_{k}$. If this is not true, there is an $f$ in $E^{\prime}$ such that $(z, f)>1$ while $|f| \leqq 1$ on $\bigcap_{k=1}^{n} a_{k} V_{k}$. As $a_{k} V_{k}$ is closed and $1 / a_{k} V_{k}^{\circ}$ is weak-star compact, the set $\left(\bigcap_{k=1}^{n} a_{k} V_{k}\right)^{\circ}$ is the convex envelope of the sets $1 / a_{k} V_{\boldsymbol{k}}^{\circ}(k=1, \cdots, n)$, which is contained in $W$. Thus $f$ is in $W$ and, since $|z| \leqq 1$ on $W,(z, f)>1$ is not possible.

Now, given $f$ in $E^{\prime}$, we can find $x_{n}$ in $\bigcap_{k=1}^{n} a_{k} V_{k}$ so that $\left|\left(f, x_{n}\right)-(z, f)\right|$ $<1 / n$. The sequence $x_{n}$ is evidently bounded in $E$, and has a weak-star cluster point $y$ in $E^{\prime \prime}$. If $m$ is any fixed integer and $n \geqq m,\left|x_{n}\right| \leqq 1$ on the 
convex envelope of $1 / a_{k} V_{\boldsymbol{k}}^{\circ}(k=1, \cdots, m)$. Therefore $|y| \leqq 1$ on the convex envelope of the same set. Because $m$ is arbitrary, $|y| \leqq 1$ on $W$; that is, $y$ is in $C$. At the same time, $(y, f)=(z, f)$. In other words $z$ and $C$ cannot be separated by a hyperplane, and $z$ must lie in the weak-star closure of $C$.

Corollary. The GF topology has a fundamental system of $T^{\prime}$ closed neighborhoods.

Proof. If $B$ is a weak-star compact set in $E^{\prime *}$ and $C$ is a bounded set in $E^{\prime \prime}$ whose weak-star closure contains $B$, then the polar of $B$ contains the polar of the weak-star closure of $C$, which is the same as the polar of $C$. The polar of $C$ is closed. In this way an arbitrary $G F$ neighborhood contains a closed one.

It is interesting to notice in this connection that the ordinary topology on the dual of any locally convex space is the strongest having a fundamental system of weak-star closed neighborhoods. For a weak-star closed convex and circled set always has the form $B^{\circ}$. If $B^{\circ}$ is to be a neighborhood, $B$ must be bounded, and $B^{\circ}$ is then an ordinary neighborhood.

As an immediate consequence of Theorem 1 we have:

Theorem 2. If the dual of $E$ is symmetric, then it is a GF dual.

We note, at this point, that A. Grothendieck has discovered an example of an $F$ space with a nonsymmetric dual [8]; hence the dual of a metric space need not be symmetric; nevertheless we do have:

THEOREM 3. If a bounded subset $B$ of $E^{\prime \prime}$ is the union of a sequence of sets $B_{n}$, each of which is bounded on a neighborhood in $E^{\prime}$, then $B$ is bounded on a neighborhood in $E^{\prime}$.

Proof. Let $c_{n}=\sup \left\{|(z, f)|: f\right.$ in $V_{n}^{\circ}, z$ in $\left.B\right\}$. By hypothesis there is a bounded set $C_{n}$ in $E$ such that $B_{n} \subseteq C_{n}^{\text {O० }} \subseteq E^{\prime \prime}$. There are numbers $a_{n k}$ such that $C_{n} \subseteq a_{n k} V_{k}$ for $k \geqq n$. Let $b_{k}=\max \left(c_{k}, a_{n k}: n \leqq k\right)$. Now, if $n \leqq k, a_{n k} \leqq b_{k}$, which implies that $a_{n k} V_{k} \subseteq b_{k} V_{k}$, so that $\bigcap_{k=n+1}^{\infty} a_{n k} V_{k} \subseteq \bigcap_{k=n+1}^{\infty} b_{k} V_{k}$, and $\left(\bigcap_{k=n+1}^{\infty} b_{k} V_{k}\right)^{\circ} \subseteq\left(\bigcap_{k=n+1}^{\infty} a_{n k} V_{k}\right)^{\circ} \subseteq C_{n}^{\circ}$. Hence $B_{n} \leqq 1$ on $\left(\bigcap_{k=n+1}^{\infty} b_{k} V_{k}\right)^{\circ}$. Also $B_{n} \leqq 1$ on $1 / b_{k} V_{k}^{\circ}$, and therefore on the convex envelope for $k=1, \cdots, n$. Since $b_{k} V_{k}$ is closed and $1 / b_{k} V_{\boldsymbol{k}}^{\circ}$ is compact, the convex envelope of $1 / b_{k} V_{\boldsymbol{k}}^{\circ}(k=1, \cdots, n)$ is $\left(\bigcap_{k=1}^{n} b_{k} V_{k}\right)^{\circ}$. Finally $B_{n} \leqq 2$ on $\left(\bigcap_{k=1}^{n} b_{k} V_{k}\right)^{\circ}$ $+\left(\bigcap_{k=n+1}^{\infty} b_{k} V_{k}\right)^{\circ}$, which contains $\left(\bigcap_{k=1}^{\circ} b_{k} V_{k}\right)^{\circ}$. The last set is a neighborhood on which all $B_{n}$ are bounded.

We conclude the discussion of metric spaces with an extension of the interior mapping theorem of $\$ 1$. A weak-star continuous transformation from $F^{\prime}$ to $E^{\prime}$ is one which is continuous when both $F^{\prime}$ and $E^{\prime}$ have the weakstar topologies.

TheOREM 4. If $E$ and $F$ are $F$ spaces and $E$ has a $G F$ dual, then every weak-star continuous linear transformation $T$ of $F^{\prime}$ onto $E^{\prime}$ is a homomorphism. 
Proof. Since the duals of $E^{\prime}$ and $F^{\prime}$ are, respectively, $E$ and $F$ when $E^{\prime}$ and $F^{\prime}$ have the weak-star topologies, the adjoint of $T$ is a continuous linear transformation $S$ from $E$ to $F$. Furthermore, $S^{*}=T$. We apply the following result of Dieudonné and Schwartz [7].

Let $E$ and $F$ be $F$ spaces and let $u$ be a continuous linear transformation from $E$ into $F$. In order that $u$ be an isomorphism of $E$ into $F$ it is necessary and sufficient that $u^{*}(F)=E^{\prime}$.

Clearly $S(E)$ is closed in $F$, for $E$ is complete and $S$ is an isomorphism. The hypotheses of the interior mapping theorem of the first section are satisfied and that theorem asserts that $T=S^{*}$ is a homomorphism.

It is a consequence of Theorem 2 that every reflexive $F$ space has a $G F$ dual. We establish the same conclusion for reflexive $L F$ spaces.

Theorem 5. Every reflexive $L F$ space $E$ has a $G F$ dual.

Proof. Let $E_{n}$ be the defining sequence of $F$ spaces and let $z$ be a bounded linear form on $E^{\prime}$. We parallel an argument of Dieudonné and Schwartz [7] to show that $z$ vanishes on some $E_{n}^{\circ}$. If it does not, there exists $f_{n}$ in $E_{n}^{\circ}$ with $\left(z, f_{n}\right)=n$. But each $x$ in $E$ is in all but a finite number of $E_{n}$ so that $\left(f_{n}, x\right)$ $=0$ for all but a finite number of $n$, and the sequence $f_{n}$ is weak-star bounded, hence bounded in $E^{\prime}$. Thus $\left(z, f_{n}\right)=n$ is impossible.

If $z$ vanishes on $E_{n}^{\circ}$, then $z$ can be regarded as a linear form on $E^{\prime} / E_{n}^{\circ}$ $=E_{n}^{\prime}$. Since $E_{n}$ is symmetric, the bounded sets in $E_{n}^{\prime}$ are the polars of neighborhoods, that is, sets of the type $\left(V \cap E_{n}\right)^{\circ}=V^{\circ}+E_{n}^{\circ}$, where $V$ is a neighborhood in $E$. As $z$ is bounded on $E^{\prime}$, it is bounded on each of these sets and is a bounded linear form on $E_{n}^{\prime}$. $E_{n}^{\prime}$, being the dual of a reflexive $F$ space, is boundedly closed, so that $z$ lies in $E_{n}$ and hence in $E$. $E^{\prime}$ is boundedly closed.

We have observed that the dual of any semi-reflexive space is symmetric. It follows that the ordinary topology of $E^{\prime}$ is a $G F$ topology. The symmetry of $E$ insures that the two natural $G F$ topologies of $E^{\prime}$ coincide and have the dual $E^{\prime *}=E$. Application of the uniqueness theorem completes the proof.

\section{BIBLIOGRAPHY}

1. L. Alaoglu, Weak topologies of normed linear spaces, Ann. of Math. vol. 41 (1940) pp. 252-267.

2. R. Arens, Duality in linear spaces, Duke Math. J. vol. 14 (1947) pp. 787-794.

3. S. Banach, Théorie des opérations linéaires, Warsaw, 1932.

4. N. Bourbaki, Sur certains espaces vectoriels topologiques, Annales de l'Institut Fourier, Université de Grenoble vol. 2 (1950) pp. 5-16.

5. J. Dieudonne, La dualité dans les espaces vectoriels topologiques, Ann. École Norm. vol. 59 (1942) pp. 108-139. $65-76$.

6. —, Une gênéralisation des espaces compact, J. Math. Pures Appl. vol. 23 (1944) pp.

7. J. Dieudonné and L. Schwartz, La dualité dans les espaces $F$ et $L F$, Annales de l'Institut Fourier, Université de Grenoble vol. 1 (1949) pp. 61-101. 
8. A. Grothendieck, Critères généraux de compacité dans les espaces vectoriels localement convexes, C. R. Acad. Sci. Paris vol. 231 (1950) pp. 940-942.

9. - Quelques résultats relatifs à la dualité dans les espaces F, C. R. Acad. Sci. Paris vol. 230 (1950) pp. 1561-1563.

10. J. Kelley, Convergence in topology, Duke Math. J. vol. 17 (1950) pp. 277-283.

11. G. Mackey, Equivalence of a problem in measure theory to a problem in the theory of vector lattices, Bull. Amer. Math. Soc. vol. 50 (1944) pp. 719-722.

12. - On convex topological linear spaces, Trans. Amer. Math. Soc. vol. 60 (1946) pp. 519-537.

13. - On infinite-dimensional linear spaces, Trans. Amer. Math. Soc. vol. 57 (1945) pp. 155-207.

14. J. von Neumann, On complete topological spaces, Trans. Amer. Math. Soc. vol. 37 (1935) pp. 1-20.

15. L. Schwartz, Théorie des distributions, Actualités Scientifiques et Industrielles, no. 1091, Paris, 1950.

16. A. Tarski, Über unerreichbare Kardinalzahlen, Fund. Math. vol. 30 (1938) pp. 68-89.

17. S. Ulam, Zur Masstheorie in der allgemeinen Mengenlehre, Fund. Math. vol. 16 (1930) pp. $140-150$.

UNIVERSITY OF WiSCONSIN, MADISON, Wis. 